\title{
Simulation of Tunable Power Multimode Interference Couplers
}

\author{
Chaojun Yan $^{1, a^{*}}$ and WenBiao Peng ${ }^{1, b}$ \\ ${ }^{1}$ College of Computer and Information technology, Three Gorges University, YiChang, China \\ a78498164@qq.com, bpwb@ctgu.edu.cn
}

Keywords: Integrated optics; Multimode interference coupler; Rib; Power tunable;3D Simulation

\begin{abstract}
The multimode interference (MMI) couplers, which operate at 1.55 microns in deep rib InGaAsP/ InP waveguide with large lateral confinement and tunable power splitting ratios, are of high interest in integrated optics. The gold contacts are applied on the top of waveguides where tuning is desired and the plasma effect will lead to negative refractive index change. The three-dimensional (3D) finite difference beam propagation method (FD-BPM) is used to model the tunable MMI couplers. In our design, the length of MMI is 180 microns. For deep rib structure, the effective index (EI) method can not be used to simplify the 3D waveguide to plane waveguide because its lower precision, and then the direct 3D FD-BPM simulation is necessary for the design of 3D MMI couplers.
\end{abstract}

\section{Introduction}

The multimode interference couplers with tunable power splitting ratios [1] are of high interest in integrated optics due to the well-defined coupler relations provided by these devices. One of the approaches to build MMI couplers with tunable power splitting ratios is to modify the material properties around certain spots [2] within the MMI where the most dominant self-images appear. For the convenience of device fabrication, the overlap-type MMI couplers [3] with deep rib structure, as showed in Fig.1, is selected to make the tunable device. The gold contacts are applied on the top of waveguides where tuning is desired and this allowed us to individually current bias the different sections. The plasma effect will lead to negative refractive index change [2]. In fact, the modulation of refractive index changes the phase relationship of these self images and finally affects the interference pattern at the output plane.

In the practical design of tunable MMI couplers, we must accurately locate the spots where the most dominant images appear and the tuning is desired. In $2 \times 2 \mathrm{MMI}$ couplers, only four self-images can be indentified halfway along the MMI coupler, so, the locations of these four self-images are the spots we seek. As showed in Fig.1 (a), two groups of spots are classified. One is called edge-biased and another is called center-biased [2]. The difference between these two type of spots exists on the different locations of gold contacts on the top of waveguide. The determination of the locations of these spots becomes the key step of an accurate design of tunable MMI couplers.

The theoretical description of the overlap-type MMI is based on the guided mode propagation analysis (MPA) method [3] which is a kind of 1D analytical approximate method and can't be used for the accurate design of 3D MMIs[4]. In paper [2], as a comparison with the MPA approximate method, the simulation of 3D beam propagation method showed the good agreement with the theoretical description. The question is that the detailed description about the 3D BPM simulation in this paper is lacking and the BPM simulation did not aim at the help of location of spots.

\section{MPA description of MMIs with tunable power}

Some formulae about the structural parameters of MMI couplers and power distribution at output guides are showed in this section and these results indicate the necessity of our modeling methods. 

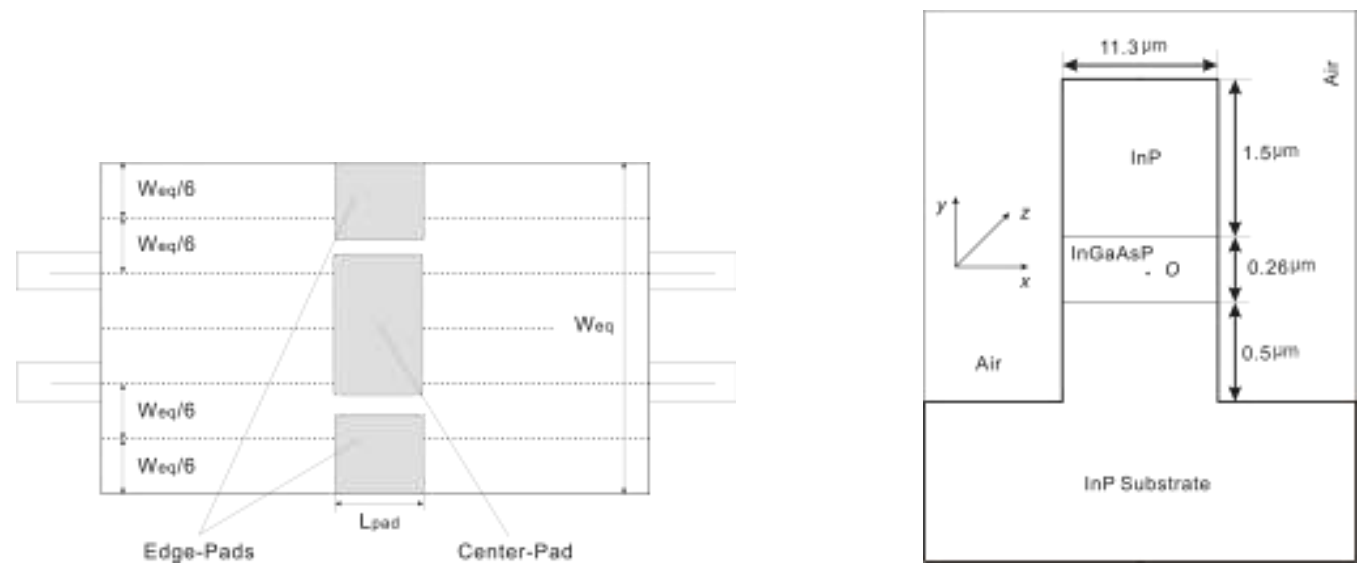

Figure 1. (a) Top view of a $2 \times 2 \mathrm{MMI}$ with locations of the pads where the negative refractive index change is induced to tune the splitting ratios. (b) Cross section of multimode waveguide with deep rib structure

As showed in Fig.1 (a), for $2 \times 2$ MMI couplers, the pads should locate in the middle of the waveguide along the direction of light propagation. So, the length of MMI couplers with $N-1$ possible input and $N-1$ output guides without index modulation must be determined. The MMI couplers with five input and output guides is considered here, namely $N=6$. If the light is only injected into input $P_{i=2}$ or $P_{i=4}$, the light from output $P_{f=2}$ and $P_{f=4}$ will be with power ratio of 50:50, and thus the MMI couplers acts as a $2 \times 2$ splitter. According to the theory of MPA about overlap-type MMI couplers,

$$
L_{N}^{M}=\frac{M}{N} \frac{4 n_{e f f}^{\prime} W_{e q}{ }^{2}}{\lambda}
$$

Where, $n_{\text {eff }}^{\prime}$ is effective refractive index and $W_{e q}$ is equivalent width of multimode waveguide. M is integer and is usually selected as one for compact device.

Now, we have a conclusion that traditional 1D analytical method is not suitable for the accurate design of tunable MMI couplers including the location of electric pads or the calculation of power splitting ratio for our case. The FD-BPM is a widely used important numerical simulation method for optical waveguide devices. The alternate directions implicit (ADI) scheme was constructed to improve computing efficiency for 3D devices. The 3D FD-BPM will be used to model the device and the detail description of this method has been illustrated in paper [5] .

\section{Modeling of tunable power MMI couplers}

The cross section of device is showed in Fig.1 (b) including the transverse dimensions of InP and InGaAsP layers. The wavelength is $1.55 \mu \mathrm{m}$. The waveguide core is the InGaAsP layer whose refractive index $n=3.38$. The $\operatorname{InP}$ layer $(n=3.17)$ and the air $(n=1)$ are used as the cladding layers.

Let's first determine the length of the $2 \times 2 \mathrm{MMI}$ couplers. If the light with amplitude function of $\exp \left\{-\left[\left(x-x_{0}\right) / 0.45\right]^{2}-\left[\left(y-y_{0}\right) / 0.45\right]^{2}\right\}$ is injected from input guide $P_{i=2}$, where $\left(x_{0}, y_{0}\right)$ is the coordinates of the center of input guide. The field profiles of four self-images at the plane of $z=L_{M M I} / 2$ and the two images at the output plane of $z=L_{M M I}$ are showed in Fig.2. The length of the $2 \times 250: 50$ splitter is determined by the quality of images through BPM simulation and the result is $L_{M M I}=180 \mu \mathrm{m}$. 


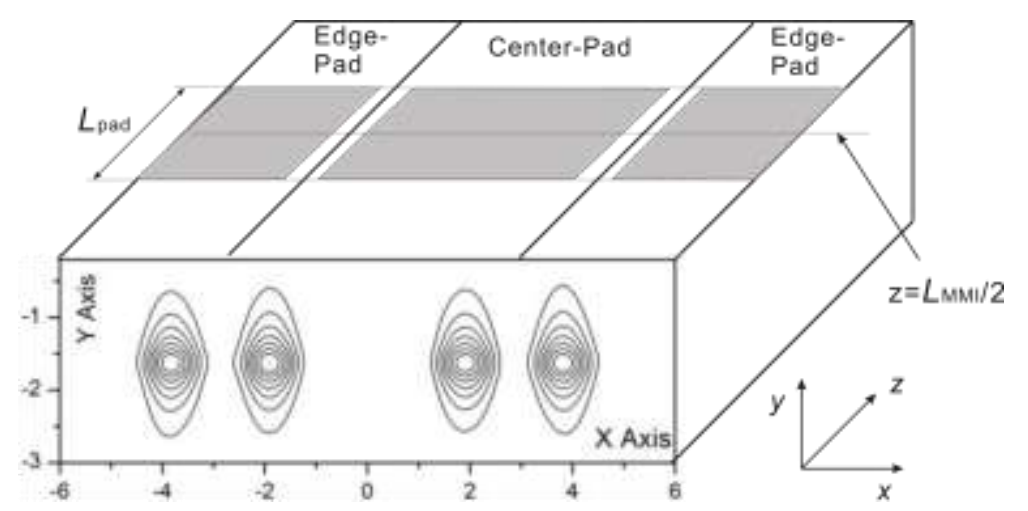

Figure 2. The locations of pads are determined by the four self-images halfway along the MMI.

If the coordinates of center of the InGaAsP layer are $O(0,-1.63)$, the positions of four self-images halfway along the MMI in x-direction are determined clearly from the Fig. 2 and they are $x_{1}=-3.812, x_{2}=-1.917, x_{3}=1.917, x_{4}=3.812$ respectively. Based on these dates, the spots where the modulation is desired are determined. The positions and the dimensions of pads on the top of waveguide are illustrated in Fig.2. For example, the range of the center-biased pad is from $x_{1}+\left(x_{2}-x_{1}\right) / 2$ to $x_{3}+\left(x_{4}-x_{3}\right) / 2$ in $\mathrm{x}$-direction as a possible selection.

Now, the tuning of MMI is considered. Firstly, $L_{p a d}$, which is the length of pad, is supposed to be $50 \mu m$ invariably. $\Delta n=b \cdot I$, with $b=-1.8 \times 10^{-4} / m A$, where $\Delta n$ is refractive index change in the guiding layer as a function of the applied current [2]. Along the direction of waveguide, the modulation area is from $z=L_{M M I} / 2-L_{p a d} / 2$ to $z=L_{M M I} / 2+L_{p a d} / 2$. When the refractive index $\Delta n$ is tuned from $0 \sim-0.027$, the normal power splitting ratio at two output waveguides, $P_{=}$and $P_{C}$, are illustrated in Fig.4. The power ratio between cross port and parallel port at output plane is tuned from 50:50 to maximums 88.5:11.4 for edge-biased. The power ratios for center-biased pad are similar with that for edge-biased pads except that the power of parallel port is gradually increased with the increasing of tuning current.

If the transverse position of pad is changed a little, or in other words, the pad can not be located accurately, can the results also be accepted? For this purpose, we have changed the range of the center-biased pad and the splitting ratios of powers at the output guides were calculated by FD-BPM. It is obvious that there exists optimal location of pad in the meaning of the most effective modulation from the data in table 1 . We recall that the pad should locate at the place where the most dominant self-images appear, so, if the pad deviates from this dominant place, the reduction of modulation efficiency can be expected. In our example, the difference between our five locations of gold pads is small but for practical application the splitting ratio corresponding to certain current of modulation must be calculated.

\section{Conclusion}

In this paper, the power tunable MMI coupler with deep rib structure is modeled by 3D FD-BPM. The length of tunable MMI is first determined by simulation, subsequently, the tuning property is modeled for two types of modulation projects. The power ratios between two output ports are accurately calculated. For deep rib structure, the effective index (EI) method can't be used to simplify the 3D waveguide to plane waveguide, so it should be emphasized here that the direct 3D FD-BPM simulation is necessary for the design of 3D MMI couplers.

\section{References}

[1] Zou Haifeng,Jiang Xiaoqing, Yang Jianyi and Wang Minghua. Analysis on Multimode Interference Coupler-Based $1 \times 3$ Optical Switch with Mono-Compound Modulation Region. 
Acta Optica Sinica, 27(2007) 1691-1694

[2] L.Juerg and H.J.Charles. Multimode interference couplers with tunable power splitting ratios. Journal of lightwave technology, 19(2001) 700-707.

[3] Sun Yiling, Jiang Xiaoqing and Wang Minghua. Analysis of general self-imaging positions in multimode interference couplers. Acta Optica Sinica, 24(2004) 759-762

[4] V. Carmen and F.H. Gil. Three-dimensional method for simulation of multimode interference couplers, Journal of lightwave technology, 13(1995) 2296-2299.

[5] Yan Chaojun,Peng Wengbiao and Li Haijun. Parallel finite difference beam propagation method based on message passing interface: application to MMI couplers with two-dimensional confinement, 5(2007) 516-518

[6] J.M. Heaton, and R.M.Jenkins. General matrix theory of self-imaging in multimode interference(MMI) couplers, IEEE Photon. Technol.Lett., 11(1999): 212-214

[7] Shi Hongwei, Wei HnogZheng, Yu JingZhong,Liu ZhongLi, $1 \times 4$ MMI splitter based on rib SOI waveguide, Chinese J Lasers B,9(2000) 525-530.

[8] Le Zichun,Hang Sungang,Hu JinHua, General self-imaging properties for line-Tapered MMI couplers , Acta Optica Sinica,31(2001) 0611003.

[9] Zhao Cezhou,Liu Enke, Fabrication of SOI single-mode rib waveguide with large trapezoidal cross-section, Acta Optica Sinica,14(1994) 783-784.

[10] Yab Chaojun,Peng wenbiao,Verification of single-mode condition of SOI rib waveguides with large cross-section, Acta Optica Sinica,32(2012) s113002. 